\title{
Inhibition of microRNA-21 upregulates the expression of programmed cell death 4 and phosphatase tensin homologue in the A431 squamous cell carcinoma cell line
}

\author{
XIAOHONG LI ${ }^{1}$, KAI HUANG ${ }^{2}$ and JIANBIN YU ${ }^{1}$ \\ Departments of ${ }^{1}$ Dermatology and ${ }^{2}$ Oncology, The First Affiliated Hospital, \\ Zhengzhou University, Zhengzhou, Henan 450052, P.R. China \\ Received September 9, 2013; Accepted March 11, 2014
}

DOI: $10.3892 / \mathrm{ol} .2014 .2066$

\begin{abstract}
RNA-21 (miRNA/miR-21) is a well-known oncogenic miRNA that is overexpressed in various carcinomas. The tumor suppressor genes, programmed cell death 4 (PDCD4) and phosphatase tensin homologue (PTEN), which target miR-21, are underexpressed in several types of cancer. However, the expression of miR-21 and its target genes, PDCD4 and PTEN, has not yet been reported in skin squamous cell carcinoma (SCC). In the present study, anti-miR-21 was transfected into the A431 cell line, and the expression of miR-21, PDCD4 and PTEN and the level of cell apoptosis were detected by quantitative polymerase chain reaction, immunocytochemistry and western blotting, and terminal deoxynucleotidyl transferase-mediated dUTP nick end labeling, respectively. The expression levels of PDCD4 and PTEN in the A431 cell line transfected with anti-miR-21 were significantly increased $(\mathrm{P}<0.05)$ and the apoptotic ratio was significantly increased $(\mathrm{P}<0.05)$. The data indicate that miR-21 may play an oncogenic role in the cellular processes of SCC and represent a novel target for effective therapies.
\end{abstract}

\section{Introduction}

Squamous cell carcinoma (SCC) is the second most common type of non-melanoma cancer of the skin. Although there are a number of associated risk factors, the etiology of this cancer has not yet been determined. microRNA-21 (miRNA/miR-21) is overexpressed in several types of solid tumors, including esophageal (1), stomach (2), colorectal (3), prostate (4), pancreatic (5), lung (6) and head and neck (7) cancers. It has been reported that the downregulation of miR-21 suppresses

Correspondence to: $\mathrm{Dr}$ Xiaohong $\mathrm{Li}$, Department of Dermatology, The First Affiliated Hospital, Zhengzhou University, 1 Jianshe East Road, Zhengzhou, Henan 450052, P.R. China

E-mail: xhhkxn@163.com

Key words: carcinoma, squamous cell, microRNA-21, programmed cell death 4 , phosphatase tensin homologue, apoptosis tumor growth and invasion in breast, glioma and colon cancer cells. Furthermore, the inhibition of miR-21 can regulate the expression of phosphatase tensin homologue (PTEN) and programmed cell death 4 (PDCD4) in cancer cells. However, the biological roles of miR-21 in SCC of the skin remain poorly understood and require further study. To the best of our knowledge, no previous studies have investigated the role of miR-21 in the A431 cell line. The present study was the first to examine the expression of PDCD4, PTEN and cell apoptosis in A431 cells transfected with anti-miR-21.

The findings of the current study demonstrate that miR-21 plays an oncogenic role in the process of SCC and may serve as a target for effective therapy of SCC of skin.

\section{Material and methods}

Cell culture. The A431 cells of human cutaneous SCC were kindly provided by the Cell Resource Center of the Institute of Basic Sciences, Chinese Academy of Medical Sciences (Tianjin, China). The cells were cultured in Dulbecco's modified Eagle's medium containing $10 \%$ fetal bovine serum (Gibco-BRL, Carlsbad, CA, USA) and incubated in a humidified atmosphere of $5 \% \mathrm{CO}_{2}$ at $37^{\circ} \mathrm{C}$. The cells were divided into three groups as follows: i) untreated A431 cells; ii) A431 cells transfected with an unrelated fragment (negative control); and iii) A431 cells transfected with antisense oligonucleotide (ASO)-miR-21.

Quantitative polymerase chain reaction ( $q$ PCR). For the A431 cells, total RNA was isolated using TRIzol reagent (Invitrogen Life Technologies, Carlsbad, CA, USA) according to the manufacturer's instructions. Total RNA ( 200 ng) was reverse transcribed using gene-specific reverse transcription primers from the TaqMan microRNA assays (Applied Biosystems, Foster City, CA, USA) and the TaqMan microRNA Reverse Transcription kit (Takara Bio, Inc., Shiga, Japan) according to the manufacturer's instructions. qPCR was performed on the iQ5 Multicolor Real-time Detection System (Bio-Rad, Hercules, CA, USA) under the following conditions: initial denaturation for $3 \mathrm{~min}$ at $95^{\circ} \mathrm{C}$, followed by 40 cycles for denaturation for $30 \mathrm{sec}$ at $95^{\circ} \mathrm{C}$, combined annealing for $30 \mathrm{sec}$ at $56^{\circ} \mathrm{C}$ and primer extension for $30 \mathrm{sec}$ at $72^{\circ} \mathrm{C}$. To estimate the expression 

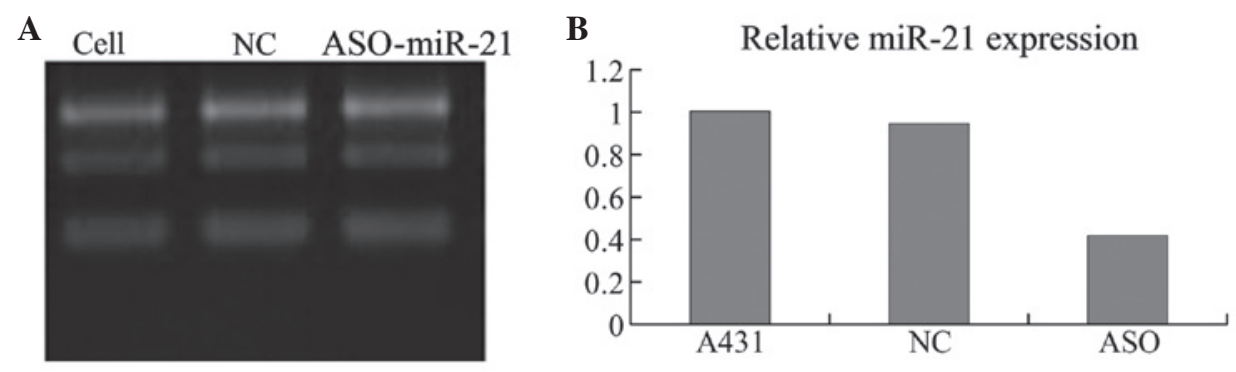

Figure 1.(A) Quantitative polymerase chain reaction (qPCR) result demonstrating mRNA expression of miR-21 in A431 cells, cells transfected with an unrelated fragment of control (NC) and cells transfected with ASO-miR-21. (B) The relative expression level of miR-21 in the A431 cells was significantly decreased by ASO-miR-21 compared with the control ( $\mathrm{P}<0.05)$. NC, normal contol; ASO, antisense oligonucleotide; miR, microRNA.

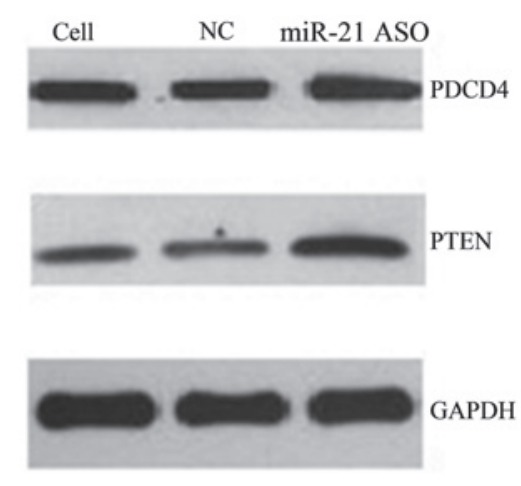

Figure 2. Western blot analysis of PDCD4 and PTEN expression in A431 cells. PDCD4 and PTEN expression in the A431 cells was evidently increased by ASO-miR-21 compared with the control ( $\mathrm{P}<0.05)$. PDCD4, programmed cell death 4; PTEN, phosphatase tensin homogue; ASO, antisense oligonucleotide; miR, microRNA; NC, negative control.

of miR-21, the Ct values were normalized using $18 \mathrm{~S}$ rRNA as an internal control. The relative miRNA expression level was calculated using the $2^{-\Delta \Delta C t}$ method. The primer for miR-21 detection was 5'-TGCGGTAGCTTATCAGACTGATG-3', and the heat shock RNA-U6 primer was 5'-TGCGGGTGCTCGCTTCGG CAGC-3'.

Transfection of ASO-miR-21. ASOs of human miR-21 were transfected by Lipofectamine ${ }^{\mathrm{TM}} 2000$ reagent (Invitrogen Life Technologies). The primer sequence of ASO was 5'-TCAACA TCAGTCTGATAAGCTA-3'.

Immunocytochemistry. PCDC4 and PTEN protein expression was detected by immunocytochemistry of streptavidin-perosidase. Following culture, the cells were grown on microscope slides and sections were deparaffinized, heated in a microwave in $0.01 \mathrm{M}$ sodium citrate buffer for antigen retrieval, treated with $3 \% \mathrm{H}_{2} \mathrm{O}_{2}$ for 10 min and rinsed in $\mathrm{H}_{2} \mathrm{O}$ and phosphate-buffered saline (PBS). The sections were blocked in 5\% goat serum in PBS, followed by incubation with the anti-PDCD4 and anti-PTEN antibodies (both Tianjin Saier Biotechology Co., Ltd., Tianjin, China). Signals were detected with 3,3'-diaminobenzidine substrate (ZSGB-Bio, Inc., Beijing, China). PDCD4 and PTEN protein expression was evaluated by integrated optical density.

Western blot analysis. The expression levels of downstream targets of human miR-21 were determined by western blot analysis. Protein from the A431 cells was extracted using radioimmunoprecipitation assay lysis buffer (Tianjin Saier Biotechology Co., Ltd.). Samples were resolved using SDS-PAGE on a $10 \%$ Tris-HCl gel and transferred to a nitrocellulose filter membrane (Millipore, Billerica, MA, USA). The membrane was probed with specific antibodies and target proteins, including GAPDH, rabbit anti-PDCD4 and rabbit anti-PTEN. Horseradish peroxidase (HRP)-conjugated secondary antibodies (goat anti-rabbit HRP) and luminal reagent were used to detect chemiluminescence. Blots were subsequently exposed to X-ray film and developed. Bands were digitally scanned and analyzed with Labworks 4.0 system (UVP, LLC, Upland, CA, USA). Western blotting of GAPDH on the same membrane was used as a loading control. The average pixel densities and band sizes in the control bands were used to normalize band density and the size of the target proteins. Target bands from the cells were directly compared.

Apoptosis assay. The three groups of cells were washed twice with $10 \mathrm{mM}$ cold PBS and resuspended in $1 \mathrm{X}$ binding buffer at a concentration of $1 \times 10^{6}$ cells $/ \mathrm{ml}$. The cells were stained with 4',6-diamidino-2-phenylindole (DAPI) and terminal deoxynucleotidyl transferase-mediated dUTP nick end labeling (TUNEL), using the FragEL ${ }^{\mathrm{TM}}$ DNA Fragmentation Detection kit (Merck KGaA, Darmstadt, Germany). The experiments were repeated at least three times. DAPI stained the nucleus of all the cells, while fluorescein stained the nucleus of the apoptotic cells. The ratio of apoptotic cell to all the cells was used to evaluate the level of cell apoptosis.

Statistical analysis. Data are expressed as the mean \pm standard deviation of three independent cell groups. The differences between groups were assessed by an unpaired two-tailed Student's t-test. $\mathrm{P}<0.05$ was considered to indicate a statistically significant difference.

\section{Results}

ASO-miR-21 downregulates miR-21 in A431 cells. qPCR analysis demonstrated that the three groups of cells had different expression levels of miR-21 ( $\mathrm{F}=107.24, \mathrm{P}<0.05)$. miR-21 was expressed at a significantly higher level in the A431 cells and cells transfected with an unrelated fragment of control compared with the cells transfected with ASO-miR-21 $(\mathrm{P}<0.05)$. However, similar levels of miR-21 

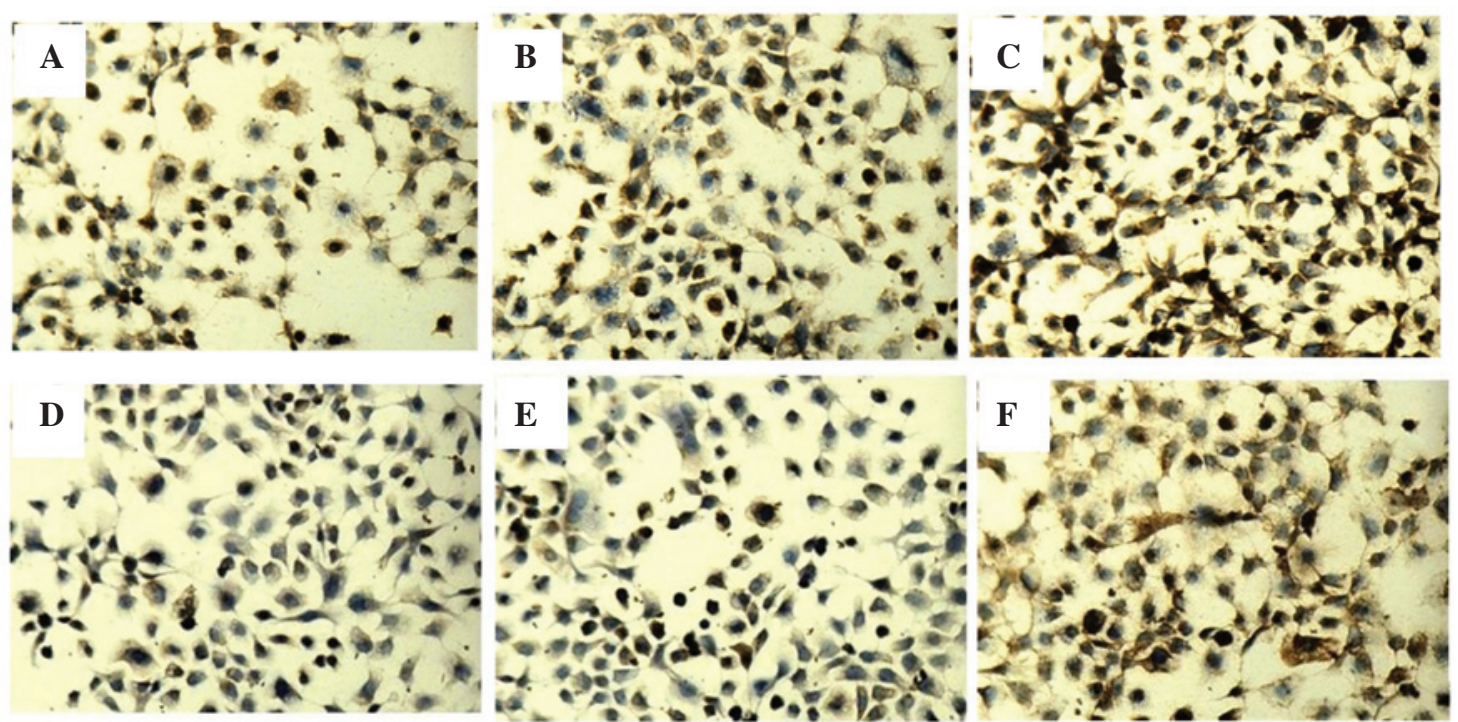

Figure 3. Expression of PDCD4 in the (A) A431 cells, (B) cells transfected with an unrelated fragment of control and (C) cells transfected with ASO-miR-21 Expression of PDCD4 in the (D) A431 cells, (E) cells transfected with an unrelated fragment of control and (F) cells transfected with ASO-miR-21. The expression of PDCD4 and PTEN in the A431 cells was evidently increased by ASO-miR-21 compared with the control, as detected by immunocytochemistry $(\mathrm{P}<0.05)$. PDCD4, programmed cell death 4; PTEN, phosphatase tensin homologue; ASO, antisense oligonucleotide; miR, microRNA.
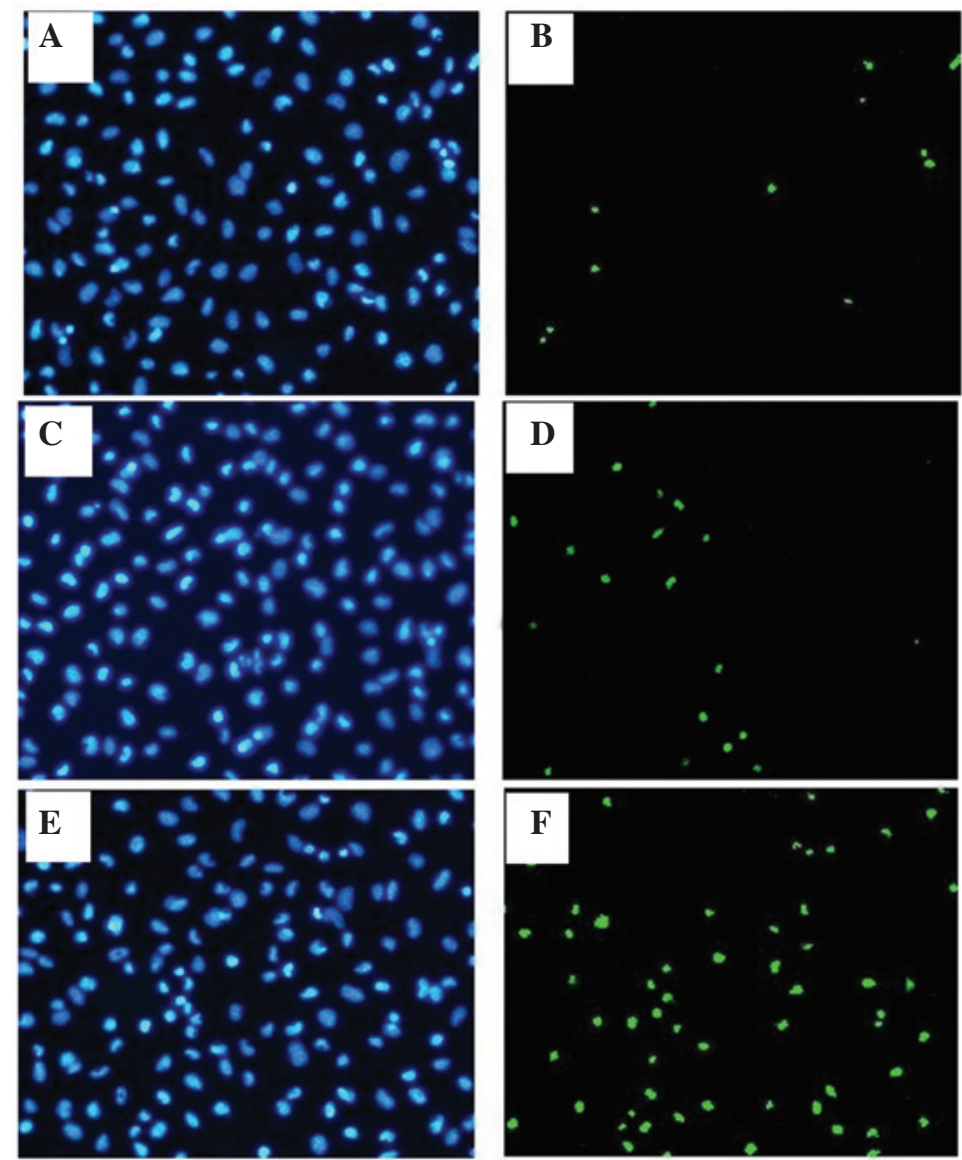

Figure 4. Apoptosis in A431 cells stained with (A) DAPI and (B) TUNEL, cells transfected with an unrelated fragment of control stained with (C) DAPI and (D) TUNEL, and cells transfected with ASO-miR-21 stained with (E) DAPI and (F) TUNEL. The apoptotic ratio in the A431 cells was evidently increased by ASO-miR-21 transfection conpared with the control group ( $\mathrm{P}<0.05)$. DAPI, 4', 6-diamidino-2-phenylindole; TUNEL, terminal deoxynucleotidyl transferase-mediated dUTP nick end labeling; ASO, antisense oligonucleotide; miR, microRNA.

expression were found between the control A431 cells and the cells without treatment $(\mathrm{P}>0.05)$. Thus demonstrating that ASO-miR-21 downregulates miR-21 in A431 cells (Fig. 1).
ASO-miR-21 upregulates the expression of PDCD4 and PTEN in A431 cells. Western blot analysis showed that the three groups of cells had different expression levels of PDCD4 
$(\mathrm{F}=11,941.13, \mathrm{P}<0.05)$ and PTEN $(\mathrm{F}=83,249.64, \mathrm{P}<0.05)$. Following ASO-miR-21 transfection, the expression of PDCD4 and PTEN in the A431 cells was evidently increased compared with the control group (Figs. 2 and 3), while similar levels of PDCD4 and PTEN expression were found between the control group and the cells without any treatment. In addition, to determine the effects of miR-21 on PDCD4 and PTEN expression, the protein levels of PDCD4 and PTEN were detected by immunocytochemistry and western blotting. The positive staining of PDCD4 and PTEN was localized in the cytoplasm and the nucleus (Fig. 3). Immunocytochemistry revealed that the three groups of cells had different expression levels of PDCD4 ( F=50.12, $\mathrm{P}<0.05)$ and PTEN $(\mathrm{F}=576.54, \mathrm{P}<0.05)$.

Downregulation of miR-21 induces apoptosis of A431 cells. To determine the effects of miR-21 on apoptosis, cell apoptosis was detected by TUNEL assay and the apoptotic ratio was used to evaluate the level of apoptosis in the cells. The three groups of cells had different apoptotic ratios $(\mathrm{F}=201.79, \mathrm{P}<0.05)$. Following ASO-miR-21 transfection, the apoptotic ratio in the A431 cells was evidently increased compared with the control group (Fig. 4), while similar apoptotic ratios were found between the control A431 cells and the cells without any treatment.

\section{Discussion}

SCC is one of the most common types of skin cancer in dermatology, and there are numerous risk factors associated with SCC of the skin, including ultraviolet-B radiation, radiation therapy, previous burns, exposure to arsenic and coal tar, human papilloma virus infection, inflammatory lesions and ulcers of long standing (8). miR-21 is overexpressed in several types of cancers and induces the invasion, intravasion and metastasis of cancer (9). PDCD4 and PTEN are the target genes of miR-21. In the present study, we hypothesized that miR-21 downregulates the expression of PDCD4 and PTEN in A431 cells, and that the inhibition of miR-21 subsequently increases PDCD4 and PTEN expression and suppresses tumor cell growth. The results showed that miR-21 affected the expression of PDCD4 and PTEN and the apoptosis of the A431 cells.

PDCD4 suppresses several targets that regulate translation and cell proliferation, and has been indicated to be involved in tissue invasion and proliferation. In a mouse cancer model, PDCD4 suppressed benign and malignant skin tumor formation and progression (10). In a study of colorectal cancer, PDCD4 mRNA levels were negatively regulated by miR-21 at each stage of cancer (11). PTEN is a phosphatase that maintains low levels of phosphatidylinositol 3,4,5-triphosphate (PIP-3) by conversion to PIP-2. When PTEN fails to maintain this homeostasis, PIP-3 levels increase and activate the protein kinase B (Akt) pathway. Activation of the Akt pathway has several effects, including the promotion of cell growth and proliferation and the inhibition of apoptosis $(12,13)$. Meng et al reported that the aberrant expression of miR-21 contributed to hepatocellular carcinoma growth and spread by modulating PTEN expression and PTEN-dependent pathways involved in mediating the cell growth, migration and invasion of cancer cells (14). Ming and He reported that PTEN negatively regulates the oncogenic phosphatidylinositol 3-kinase/Akt signaling pathway and showed that PTEN is a critical tumor suppressor for skin cancer in humans and in mice (15). A previous study of gastric cancer indicated that miR-21 inhibition may upregulate the PTEN expression level, and that the downregulation of miR-21 exhibits a stronger inhibitory effect on the biological behavior of cancer cells (16).

In the present study, ASO-miR-21 was efficiently transfected into the A431 cells resulting in a marked downregulation of miR-21 in vitro. Previous studies have shown that transfection of anti-miR-21 can downregulate miR-21 expression of oral SCC (17) and laryngeal SCC (18). In the present study, immunocytochemistry and western blot analysis revealed a significant increase in the expression of PDCD4 and PTEN in the A431 cell line transfected with anti-miR-21. Previous studies have shown that the inhibition of miR-21 in cancer cells increases PTEN and PDCD4 protein levels in HeLa and MCF-7/ADR cells $(19,20)$. Furthermore, in the present study, the TUNEL assay showed a significant increase in the apoptotic ratio in the A431 cell line transfected with anti-miR-21. miR-21 has also been found to be upregulated in laryngeal carcinoma tissues, and the knockdown of miR-21 by a specific ASO inhibited the proliferation potential of Hep-2 cells (21).

In conclusion, the present study demonstrated that miR-21 downregulates the expression of PDCD4 and PTEN, and that the inhibition of miR-21 suppresses tumor growth and invasion. Considering that PDCD4 and PTEN function as tumor suppressor genes and are the target genes of miR-21, ASO-miR-21 may have potential applications as a therapeutic target. Understanding the complex regulation of miR-21 with regard to its target gene expression in SCC may be valuable for exploring potential therapeutic methods for SCC, and gene therapy targeting miR-21 should be further investigated as a potential alternative strategy for SCC therapy.

\section{References}

1. Zhu L, Yan W, Rodriguez-Canales J, et al: MicroRNA analysis of microdissected normal squamous esophageal epithelium and tumor cells. Am J Cancer Res 1: 574-584, 2011.

2. Motoyama K, Inoue H, Mimori K, et al: Clinicopathological and prognostic significance of PDCD4 and microRNA-21 in human gastric cancer. Int J Oncol 36: 1089-1095, 2010.

3. Chang KH, Miller N, Kheirelseid EA, et al: MicroRNA-21 and PDCD4 expression in colorectal cancer. Eur J Surg Oncol 37: 597-603, 2011.

4. Ribas J and Lupold SE: The transcriptional regulation of miR-21, its multiple transcripts, and their implication in prostate cancer. Cell Cycle 9: 923-929, 2010.

5. Giovannetti E, Funel N, Peters GJ, et al: MicroRNA-21 in pancreatic cancer: correlation with clinical outcome and pharmacologic aspects underlying its role in the modulation of gemcitabine activity. Cancer Res 70: 4528-4538, 2010.

6. Wei J, Gao W,Zhu CJ, et al: Identification of plasma microRNA-21 as a biomarker for early detection and chemosensitivity of non-small cell lung cancer. Chin J Cancer 30: 407-414, 2011.

7. Tran N, Mclean T, Zhang X, et al: MicroRNA expression profiles in head and neck cancer cell lines. Biochem Biophys Res Commun 358: 12-17, 2007.

8. LeBiot PE, Burg G, Weedon D and Sarasin A: World Health Organization Classification of Tumours. Pathology and Genetics of Skin Tumours. IARC Press, Lyon, 2006.

9. Asangani IA, Rasheed SA, Nikolova DA, et al: MicroRNA-21 (miR-21) post-transcriptionally downregulates tumor suppressor Pdcd4 and stimulates invasion, intravasation and metastasis in colorectal cancer. Oncogene 27: 2128-2136, 2008. 
10. Jansen AP, Camalier CE and Colburn NH: Epidermal expression of the translation inhibitor programmed cell death 4 suppresses tumorigenesis. Cancer Res 65: 6034-6041, 2005.

11. Horiuchi A, Linuma H, Akahane T, et al: Prognostic significance of PDCD4 expression and association with microRNA-21 in each Dukes' stage of colorectal cancer patients. Oncol Rep 27: 1384-1392, 2012.

12. Altomare DA and Testa JR: Perturbations of the AKT signaling pathway in human cancer. Oncogene 24: 7455-7464, 2005.

13. Wen YG, Wang Q, Zhou CZ, et al: Mutation analysis of tumor suppressor gene PTEN in patients with gastric carcinomas and its impact on PI3K/AKT pathway. Oncol Rep 24: 89-95, 2010.

14. Meng F, Henson R, Wehbe-Janek H, et al: MicroRNA-21 regulates expression of the PTEN tumor suppressor gene in human hepatocellular cancer. Gastroenterology 133: 647-658, 2007.

15. Ming $\mathrm{M}$ and He YY: PTEN: new insights into its regulation and function in skin. J Invest Dermatol 129: 2109-2112, 2009.

16. Zhang BG, Li JF, Yu BQ, et al: MicroRNA-21 promotes tumor proliferation and invasion in gastric cancer by targeting PTEN. Oncol Rep 27: 1019-1026, 2012
17. Reis PP, Tomenson M, Cerciqne NK, et al: Programmed cell death 4 loss increased tumor cell invasion and is regulated by miR-21 in oral squamous cell carcinoma. Mol Cancer 9: 238, 2010.

18. Ren J,Zhu D, Liu M, et al: Downregulation of miR-21 modulates Ras expression to promote apoptosis and suppression invasion of laryngeal squamous cell carcinoma. Eur J Cancer 46: 3409-3416, 2010.

19. Yao Q, Xu H, Zhang QQ, et al: MicroRNA-21 promotes cell proliferation and down-regulates the expression of programmed cell death 4 (PDCD4) in HeLa cervical carcinoma cells. Biochem Biophys Res Commun 388: 539-542, 2009.

20. Wang ZX, Lu BB, Wang H, et al: MicroRNA-21 modulates chemosensitivity of breast cancer cells to doxorubicin by targeting PTEN. Arch Med Res 42: 281-290, 2011.

21. Liu M, Wu H, Liu T, et al: Regulation of the cell cycle gene, BTG2, by miR-21 in human laryngeal carcinoma. Cell Res 19: 828-837, 2009. 